\title{
COKRIGAGEM NA DISTRIBUIÇÃO ESPACIAL DO CÁLCIO BASEADO NO pH EM UM LATOSSOLO CULTIVADO COM CAFÉ CONILON
}

COSTA, Felipe Pianna ${ }^{1}$ LIMA, Julião Soares de Souza ${ }^{1}$

RESUMO: A agricultura de precisão utiliza técnicas que permite mapear a variabilidade espacial de atributos do solo, com uma análise detalhada dos atributos químicos do solo. Por esse motivo, o objetivo deste trabalho foi investigar a eficiência do método interpolação da cokrigagem na estimativa do cálcio de acordo com o pH do solo, em um área cultivada com café conilon. Os resultados mostraram uma correlação significativa de 0,83 do pH com o cálcio no solo. A cokrigagem permitiu estimar a variabilidade espacial do cálcio com precisão, apresentando mapas similares quanto à forma de distribuição espacial do atributo.

Palavras chaves: Geoestatística. Agricultura de precisão. Interpolação.

\section{COKRIGING APLLIED ON THE SPATIAL DISTRIBUTION OF CALCIUM BASED IN pH TO OXISOL ON COFFEA CANEPHORA CROP}

SUMMARY: The precision agriculture uses techniques that allows map the spatial variability of soil properties, with a detailed analysis of soil chemical properties. The aim of this study was to evaluate the eficient of mapping the chemical attribute $\mathrm{Ca}$ cokriging by using the $\mathrm{pH}$ as auxialiary variable in the estimation at an area cultivated with coffee conilon. The results showed a significant correlation 0,83 shared with $\mathrm{pH}$ and $\mathrm{Ca}$ in the soil. The cokriging has a good performance to estimation the spatial variability of Ca showing common maps for a spatial distribution of that attribute.

Keywords: Geoestatistic. Precision agriculture. Interpolation.

\section{INTRODUÇÃO}

Devido a diversos fatores de formação do solo, que envolve desde as ações realizadas por microorganismo a modificações pelo clima, os atributos do solo que se relacionam com a nutrição das plantas, apresentam variabilidade espacial. No entanto, a agricultura atual, em algumas regiões no Brasil, não tem considerado a variabilidade espacial do solo, utilizando práticas de manejo homogêneas.

A carência de uma legislação disciplinada com regras fiscalizadoras para a aplicação dos insumos agrícolas na agricultura brasileira favorece ainda mais para o quadro de escassez de bens essenciais na racionalização da aplicação de insumos agrícolas. Desta forma, a visão de curto prazo aliada a outros fatores como o foco na demanda de mercado e a rentabilidade

\footnotetext{
${ }^{1}$ Centro de Ciências Agrárias da Universidade Federal do Espírito Santo - CCA-UFES, Alto Universitário, Cx.P.16, CEP: 29500-000, Alegre-ES, felipepianna@gmail.com ; limajss@yahoo.com.br .
} 
imediata, têm contribuído para ações que canalizam para conseqüências ambientais preocupantes.

Assim, o mapeamento dos atributos do solo é importante para o manejo adequado dos nutrientes na cultura do café conilon, permitindo a visualização de áreas com déficit e excesso de nutrientes. A disponibilidade dos nutrientes para as plantas está estreitamente relacionada com a acidez do solo, que é corrigida com a realização da calagem.

Para identificar a variabilidade espacial dos atributos do solo diversas ferramentas têm sido utilizadas, dentre elas destaca-se a Geoestatística que é fundamentada na Teoria das Variáveis Regionalizadas, que, segundo Clark (1979), a distribuição das diferenças de variáveis entre dois pontos amostrados é a mesma para toda a área, e que isto depende somente da distância entre eles e da orientação dos pontos.

A utilização da geoestatística com a interpolação por meio da cokrigagem, no momento em que uma das variáveis não foi amostrada em quantidade suficiente, por dificuldades experimentais ou altos custos, pode proporcionar estimativas de precisão aceitável. Segundo Nielsen \& Wendroth (2003) os diversos atributos do solo podem ser estimados a partir de variáveis de determinação mais simples, as quais são denominadas covariáveis ou variáveis preditivas.

A cokrigagem é um método de interpolação que deriva da krigagem, já que identifica a relação de variabilidade espacial entre duas variáveis distintas (VIEIRA apud NOVAIS; ALVAREZ; SCHAEFER, 2000). Segundo Vauclin et al. (1983) a cokrigagem pode ser mais precisa do que a krigagem se a variável primária e a covariável apresentarem dependência espacial, segundo a informação gerada pelo semivariograma cruzado.

No suprimento da quantidade adequada dos nutrientes essenciais para o aumento da produtividade do café conilon é importante correlacionar os atributos de forma a minimizar a acidez do solo. Diante do exposto, o presente trabalho teve por objetivo o mapeamento da distribuição espacial do atributo cálcio utilizando como covariável o pH, segundo a técnica de cokrigagem.

\section{MATERIAL E MÉTODOS}

O trabalho foi realizado em uma área cultivada com café conilon, localizada em Cachoeiro de Itapemirim - ES nas latitude de $20^{\circ} 45^{\prime} 17,31^{\prime \prime} \mathrm{S}$ e longitude de $41^{\circ} 17^{\prime} 8,86^{\prime \prime} \mathrm{W}$. A região em estudo apresenta clima tropical (Aw), pelo sistema de Köppen, com duas estações bem definidas, sendo uma com temperaturas mais elevadas e chuvosa (outubro a abril) e outra com temperaturas mais amenas e seca ( maio a setembro ), e precipitação total 
anual média de $1.346 \mathrm{~mm}$. A área em estudo possui topografia levemente inclinada com topo arredondado.

Foram estabelecidos pontos amostrais compostos por cinco plantas espaçadas em 2,9 x $0.9 \mathrm{~m}$, gerando uma célula experimental de $13,05 \mathrm{~m}^{2}$. As amostras de solo foram retiradas na projeção da copa, totalizando 109 pontos amostrais georreferenciados compondo uma área de 1 ha. Em cada célula amostral, foram retiradas amostras de solo na profundidade de 0,0 0,20 m para a determinação dos valores do pH em água e da concentração de cálcio trocável no solo.

A primeira fase de análise dos resultados consistiu a uma análise descritiva exploratória e também a análise de correlação para determinar a relação entre as variáveis. Para a verificação de candidatos a valores discrepantes, chamados de "outliers", foram analisados os quartis superiores e inferiores e a hipótese de normalidade dos dados foi avaliada pelo teste $\mathrm{W}$ a $5 \%$. Em seguida realizou-se analise geoestatística, dentro da qual se buscou verificar a existência e quantificar o grau de dependência espacial dos atributos estudados, utilizando-se o programa $\left(\mathrm{GS}^{+}\right)$no qual foi ajustado um modelo de semivariograma cruzado, conforme a equação (1) abaixo:

$$
\gamma_{1,2}(h)=\frac{1}{2 n} \sum_{i=1}^{n}\left\{\left\{\left[z^{1}\left(x_{i}\right)-z^{1}\left(x_{i}+h\right)\right] *\left[z^{2}\left(x_{i}\right)-z^{2}\left(x_{i}+h\right)\right]\right\}\right.
$$

em que,

Equação(1)

$\gamma_{1,2}(h)$ - é o semivariograma cruzado entre a variável primária e a secundária; $z^{\mathbf{2}}\left(x_{i}\right)$ - é o valor da variável primária no ponto $\mathrm{x}_{\mathrm{i}}$;

$z^{i}\left(x_{i}+h\right)-$ é o valor da variável primária no ponto $x_{i}$ adicionado de uma distância $h$; $z^{2}\left(x_{i}\right)-$ é o valor da variável secundária no ponto $\mathrm{x}_{\mathrm{i}}$;

$z^{2}\left(x_{i}+h\right)-$ é o valor da variável secundária no ponto $x_{i}$ adicionado de uma distância $h$; $n$ - é o número de pares de pontos formados para uma dada distância $h$.

$\mathrm{Na}$ seleção dos modelos dos semivariogramas foram considerados os valores de $\mathrm{R}^{2}$ (coeficiente de determinação) e SQR (soma dos quadrados dos resíduos), segundo Robertson (1998). Para estimar valores dos atributos químicos em locais não amostrados e confeccionar os mapas temáticos para o atributo $\mathrm{Ca}$, utilizou-se a krigagem ordinária e a cokrigagem. $\mathrm{Na}$ aplicação da cokrigagem, o atributo selecionado como variável primária foi os valores das concentrações de cálcio no solo, pois segundo Cantarella et al. (apud RAIJ et al., 2001), quando a determinação de Ca é feita utilizando o sistema de extração de cátions trocáveis com solução extratora de $\mathrm{KCl} 1 \mathrm{~mol}$ L-1, através da espectrometria de absorção atômica, 
ocorrem dificuldades operacionais decorrentes da formação de depósitos de $\mathrm{KCl}$ na fenda do queimador do equipamento, onerando e tornando a análise demorada. Como covariável foi o pH em água, pelo fato de o método apresentar baixo custo e ser de rotina na maioria dos laboratórios de análises de solo. O resultado final são mapas temáticos da krigagem e da cokrigagem do atributo $\mathrm{Ca}$, tendo como covariável o $\mathrm{pH}$, gerados no programa Surfer.

\section{RESULTADOS E DISCUSSÃO}

A Figura 1 e 2 apresenta a distribuição de freqüência do atributo cálcio e pH, respectivamente, na área de estudo.

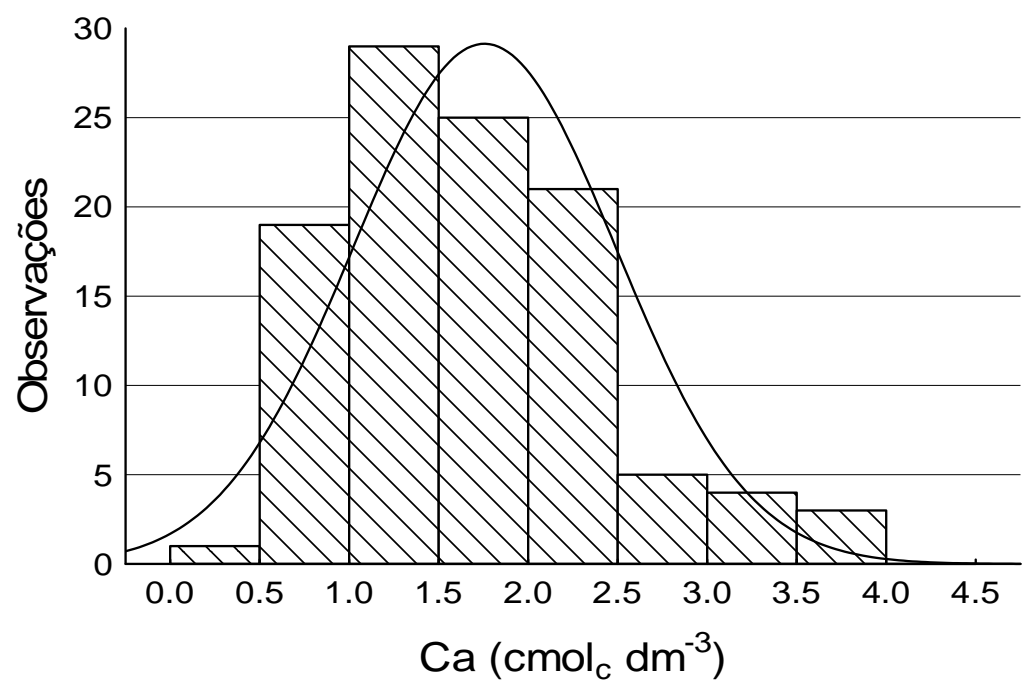

Figura 1 - Distribuição de freqüência das observações do atributo cálcio.

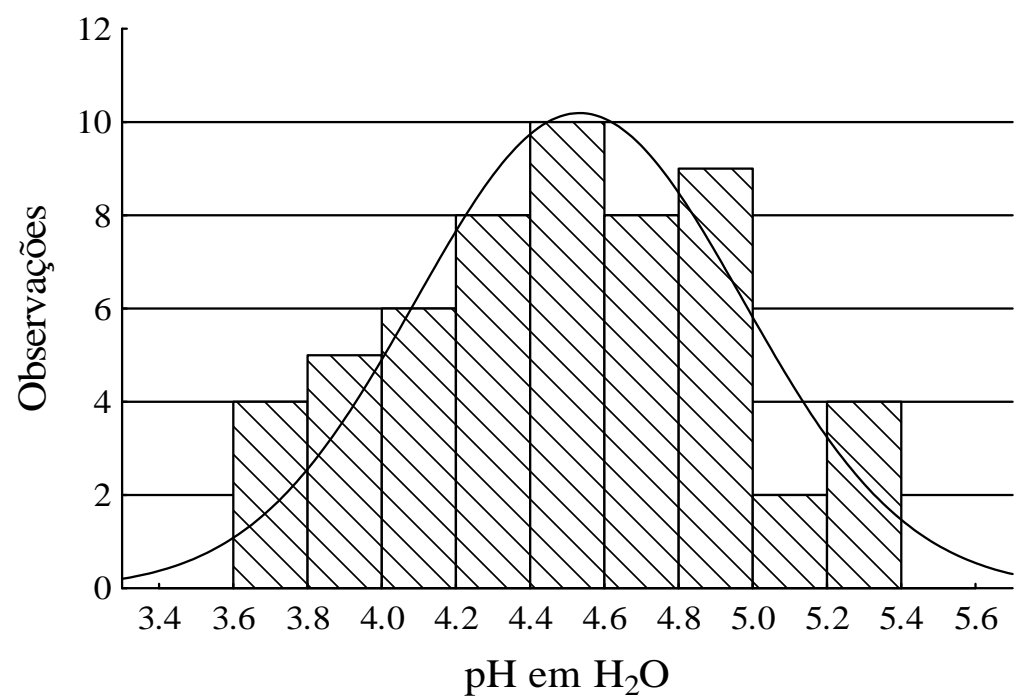

Figura 2 - Distribuição de frequiência das observações do atributo pH.

Segundo Guimarães (2005), o histograma permite a visualização do comportamento do atributo em estudo, com relação à tendência de concentração de dados (tendência simétrica 
ou assimétrica). A distribuição de frequência dos atributos químicos analisados na área de estudo, indicam assimetria à direita, com concentração de valores abaixo da média.

A análise descritiva para os atributos analisados está na Tabela 1, sem a presença dos valores discrepantes chamados de outliers.

Tabela 1. Estatística descritiva dos atributos analisados na profundidade de 0,0-0,2 m.

\begin{tabular}{llllllllllll}
\hline & & & \multicolumn{4}{c}{ Valores } & \multicolumn{4}{c}{ Coeficientes } & \multicolumn{3}{c}{ Testes } \\
\cline { 7 - 13 } Atributo & Média & Md. & $\mathrm{s}$ & $\mathrm{Q}_{1}$ & $\mathrm{Q}_{3}$ & Mín. & Máx. & $\mathrm{CV}$ & $\mathrm{C}_{\mathrm{k}}$ & $\mathrm{C}_{\mathrm{s}}$ & $\mathrm{W}$ \\
\hline $\mathrm{pH}$ & 4,6 & 4,5 & 0,6 & 4,1 & 5,0 & 3,7 & 6,3 & 13,0 & 0,3 & 0,8 & $* *$ \\
$\mathrm{Ca}$ & 1,7 & 1,6 & 0,8 & 1,3 & 2,3 & 0,4 & 3,8 & 45,0 & 1,6 & 1,1 & $* *$ \\
\hline
\end{tabular}

Md- mediana; s- desvio padrão; $\mathrm{Q}_{1}-$ valor do $1^{\circ}$ quartil ; $\mathrm{Q}_{3}-$ valor do $3^{\circ}$ quartil; Min.- valor mínimo; Max.valor máximo; $\mathrm{CV}$ - coeficiente de variação $(\%) ; \mathrm{C}_{\mathrm{k}}$ - coeficiente de curtose; $\mathrm{C}_{\mathrm{s}}$ - coeficiente de assimetria; ** distribuição normal pelo teste Shapiro-Wilks a 5\% de probabilidade.

Conforme Libardi et al. (1996), a semelhança entre as medidas de posição (média e mediana) sugerem um possível ajuste dos dados à distribuição normal, confirmado pelo teste de Shapiro-Wilk's (W) a 5\% de probabilidade. Os valores de assimetria e curtose, próximos de zero, indicam a proximidade entre a distribuição de frequiências dos dados e a distribuição normal. Segundo Paz-Gonzalez; Castro e Vieira (2001), a estimativa por krigagem de valores não medidos apresenta melhores resultados quando os dados apresentam distribuição normal.

$\mathrm{O}$ pH do solo apresentou baixo CV (13\%) por ser medido em escala logarítmica, entretanto o Ca apresentou um CV (45\%) alto segundo Pimentel-Gomes (1984).

Observa-se na Figura 3 uma alta correlação $(r=0,83)$ do pH com o Ca. Tal resultado garante a utilização da cokrigagem na estimativa de valores não amostrados para o atributo $\mathrm{Ca}$, tendo o $\mathrm{pH}$ como covariável na estimativa.

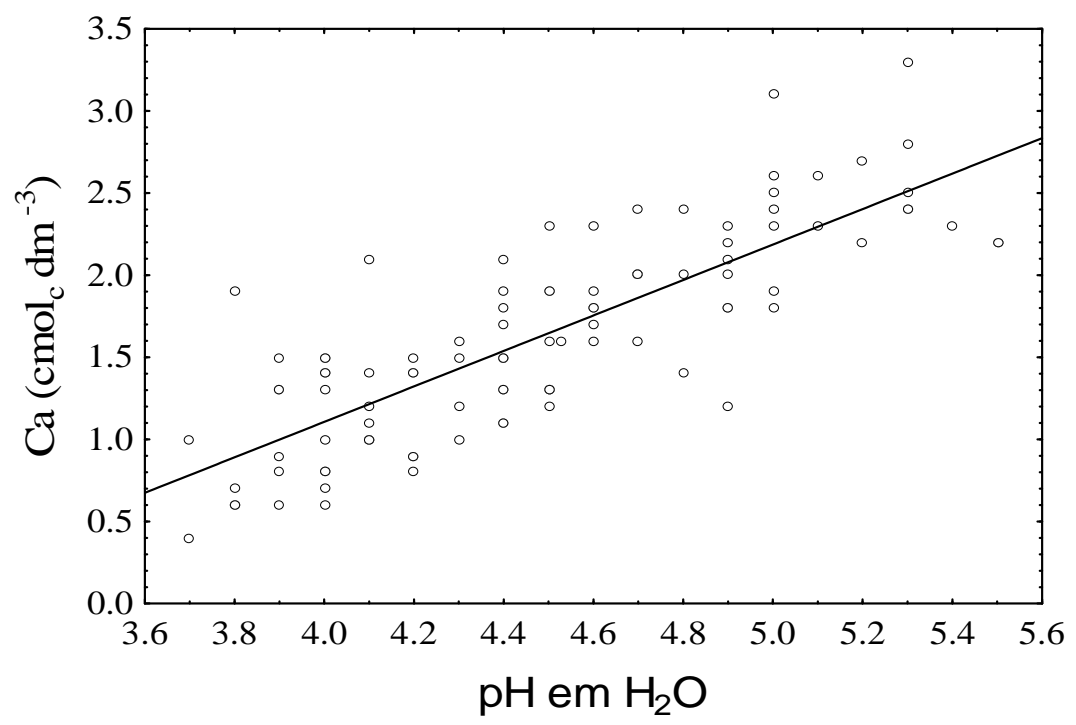

Figura 3 - Diagrama de dispersão entre os valores de pH e Ca.

A análise de correlação mostra que o atributo Ca pode ser representado pelo $\mathrm{pH}$ sem muita perda de informação e com boa precisão. A correlação entre as variáveis é um dos 
pontos necessários para a realização da cokrigagem (VIEIRA apud NOVAIS; ALVAREZ; SCHAEFER, 2000). A outra exigência é que exista dependência espacial para os atributos em estudo, conforme se observa na Tabela 2.

O modelo esférico foi ajustado aos dados do semivariograma experimental para o atributo cálcio, $\mathrm{pH}$ e na análise da cokrigagem com o semivariograma cruzado. Os alcances de dependência espacial foram de 9,5, 10,0 e 9,0 m para o cálcio, $\mathrm{pH}$ e relação pH x Ca, respectivamente. Em uma análise realizada por Silva (2009) a relação pH x Ca para o café arábica em área de montanha, num Latossolo Vermelho Amarelo húmico, encontrou um alcance de dependência espacial de 13 metros, com ajuste ao modelo esférico.

Tabela 2. Parâmetros dos semivariogramas ajustados.

\begin{tabular}{llllllr}
\hline Atributo & Modelo & $\begin{array}{c}\mathrm{a} \\
(\mathrm{m})\end{array}$ & $\mathrm{C}_{0}$ & $\mathrm{C}_{0}+\mathrm{C}$ & $\begin{array}{c}\mathrm{IDE} \\
(\%)\end{array}$ & $\begin{array}{c}\mathrm{R}^{2} \\
(\%)\end{array}$ \\
\hline $\mathrm{Ca}$ & $\mathrm{ESF}$ & 9.5 & 0,1 & 0,67 & 99,0 & 85,7 \\
$\mathrm{pH}$ & $\mathrm{ESF}$ & 10,0 & 0,1 & 0,37 & 78,0 & 84,0 \\
$\mathrm{pH} \times \mathrm{Ca}$ & $\mathrm{ESF}$ & 9,0 & 0,1 & 0,45 & 99,8 & 84,0 \\
\hline
\end{tabular}

ESF- modelo esférico; a - alcance $(\mathrm{m}) ; \mathrm{C}_{0}$ - efeito pepita; $\mathrm{C}_{0}+\mathrm{C}-$ patamar; IDE - índice de dependência espacial $\left[\mathrm{C} /\left(\mathrm{C}_{0}+\mathrm{C}\right)\right]$ e $\mathrm{R}^{2}$ - coeficiente de determinação do modelo do semivariograma.

Os alcances obtidos para todos os atributos estudados são próximos, com o mesmo modelo de ajuste de semivariograma, indicando assim o mesmo padrão de distribuição espacial.

Os atributos analisados apresentaram forte dependência espacial com IDE $\geq 75 \%$, segundo Zimback (2001). Todos os atributos apresentaram valores baixo de efeito pepita $\left(\mathrm{C}_{0}\right)$, indicando que a escala de amostragem foi suficiente para mostrar a variabilidade natural do fenômeno.

Considerando os modelos e parâmetros ajustados para os semivariogramas médios, os dados de Ca foram submetidos à interpolação pelo método da krigagem ordinária (KO) (Figura 4) e a relação pH x Ca pela cokrigagem (CK) (Figura 5) a fim de mapear a sua distribuição espacial. Analisando o mapa gerado pela KO e o mapa da CK, observa-se que estes apresentaram similaridade quanto à forma de distribuição de zonas de manejo, o que pode ser observado na Tabela 3 na porcentagem da área ocupada por cada classe, onde se verifica que o valor médio de $1,7 \mathrm{cmol}_{\mathrm{c}} \mathrm{dm}^{-3}$ de cálcio se encontra na classe 2 . 
Tabela 3. Porcentagem da área de estudo ocupada pelas diferentes classes de distribuição de cálcio pelo dois métodos de interpolação krigagem ordinária e cokrigagem.

\begin{tabular}{cccccc}
\hline \multicolumn{5}{c}{ Classes $\left(\mathrm{cmol}_{\mathrm{c}} \mathrm{dm}^{-3}\right)$} \\
& 1 & 2 & 3 & 4 & 5 \\
& $0-0,9$ & $0,9-1,8$ & $1,8-2,7$ & $2,7-3,6$ & $>3,6$ \\
\hline KO & 1 & 41 & 49 & 8 & 1 \\
CK & 1 & 44 & 43 & 10 & 2 \\
\hline
\end{tabular}

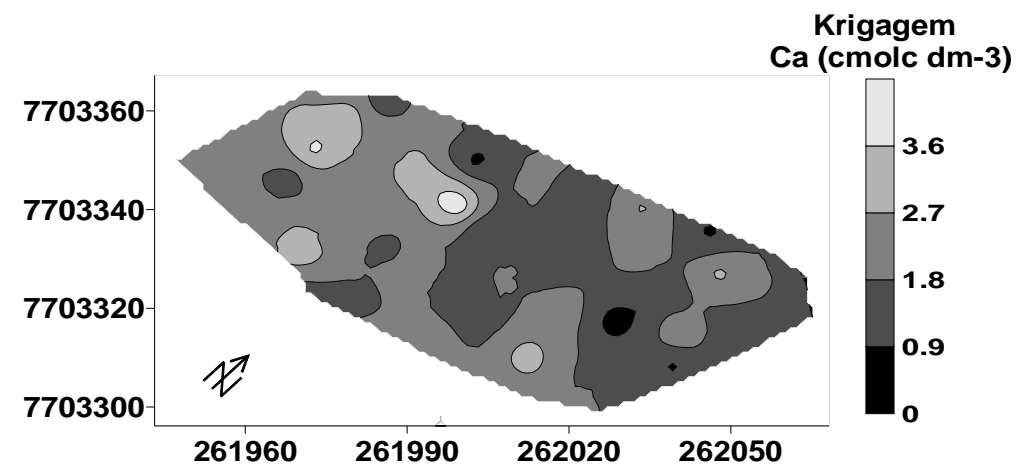

Figura 4 - Mapa da distribuição espacial do atributo Ca no solo utilizando a KO.

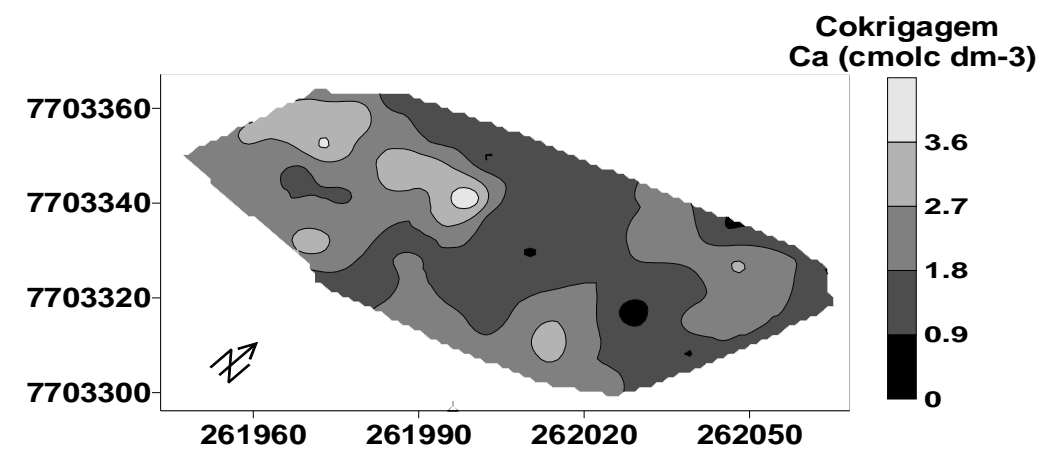

Figura 5 - Mapa da distribuição espacial do atributo Ca no solo utilizando a CK.

Dessa forma, o pH da área estudada pode ser utilizado para estimar a distribuição espacial do cálcio, conforme a similaridade apresentada entre os mapas.

\section{CONCLUSÃO}

Os atributos $\mathrm{Ca}$ e pH apresentaram alta correlação e dependência espacial na área, mostrando que a técnica de cokrigagem é um método de interpolação eficiente para estimar com precisão a distribuição espacial do cálcio.

\section{AGRADECIMENTOS}

A FAPES pelo apoio e incentivo financeiro a pesquisa. 


\section{REFERÊNCIAS}

CANTARELLA, H.et al. Determinação de alumínio, cálcio e magnésio trocáveis em extrato de cloreto de potássio. In: RAIJ, B. van et al. (eds.). Análise química para avaliação da fertilidade em solos tropicais. Campinas: Instituto Agronômico, 2001. 285p.

CLARK, I. The semivariogram - Part I. Eng. \& Min. J., v. 180, n.7, p.90-94, 1979.

GUIMARÃES, E. C. Geoestatística básica e aplicada. Uberlândia: UFU, 75p., 2005.

Disponível em: http://www.ufu.famat.br/ ednaldo.htm. Acesso em 5 jul. 2010.

LIBARDI, P.L.et al. Variabilidade da umidade gravimétrica de um solo hidromórfico. Revista Brasileira de Ciência do Solo. Campinas, v.20, p.1-12, 1996.

NIELSEN, D.R. ; WENDROTH, O. Spatial and temporal statistics: sampling field soils and their vegetation. Reiskirchen, Catena Verlag, 2003. 398p.

PAZ-GONZALEZ, A.; CASTRO, M. T.; VIEIRA, S. R. Geostatistical analysis of heavy metals in a one-hectare plot under natural vegetation in a serpentine area. Canadian Journal of Soil Science, Ottawa, v. 81, p. 469-479, 2001.

PIMENTEL-GOMES, F. A estatística moderna na pesquisa agropecuária. Piracicaba: POTAFOS - Associação Brasileira para Pesquisa da Potassa e do Fosfato, 1984. 160p.

ROBERTSON, G.P. GS ${ }^{+}$Geostatistics for the environmental sciences: GS+ user's guide. Plainwell: Gamma Design Soffware, 1998. 152p.

SILVA, S.A.et al. Cokrigagem aplicada ao mapeamento de atributos químicos do solo em Reduto-Mg - SP. In: SIMPÓSIO DE GEOESTATÍSTICA APLICADA EM CIÊNCIAS

AGRÁRIAS, 2009, Botucatu. Resumos expandidos... Botucatu: Fundação de Estudos e Pesquisas Agrícolas e Florestais, 2009. 1 CD-ROM.

VAUCLIN, M.et al. The use of cokriging with limited field soil observations. Soil Science Society of America Journal, v.47, n.1, p.175-184, 1983.

VIEIRA, S.R. Geoestatística em estudos de variabilidade espacial do solo. In: NOVAIS, R.F.; ALVAREZ, V.H.; SCHAEFER, C.E.G.R. (Eds.) Tópicos em ciência do solo. Viçosa: Sociedade Brasileira de Ciência do Solo, v.1, p.1-54, 2000.

ZIMBACK, C.R.L. Análise espacial de atributos químicos de solos para fins de mapeamento da fertilidade. 2001. 114 f. Tese (Livre-Docência em Levantamento do Solo e Fotopedologia) - Faculdade de Ciências Agronômicas, Universidade Estadual Paulista, Botucatu. 